\title{
Partial Unilateral Lentiginosis Treated with 532-nm and Subsequent Low-Fluence 1,064-nm 0-Switched Neodymium-Doped Yttrium Aluminum Garnet Lasers
}

\author{
Jiehoon Kim ${ }^{1}$ \\ Dai Hyun Kim² \\ Seung Hyun Chun ${ }^{3}$ \\ Hwa Jung Ryu ${ }^{3}$
}

${ }^{1}$ Dr. Kim's Skin \& Laser Clinic, Dermatology, Suwon, Korea

${ }^{2}$ Department of Anatomy, Korea University College of Medicine, Seoul, Korea

${ }^{3}$ Department of Dermatology, College of Medicine, Korea University Ansan Hospital, Ansan, Korea
Received June 20, 2016

Accepted June 20, 2016

\footnotetext{
Correspondence

Hwa Jung Ryu

Department of Dermatology, Korea University Ansan Hospital, 123, Jeokgeum-ro, Danwon-gu, Ansan 15355, Korea

Tel: +82-31-412-5186

Fax: +82-31-412-4208

E-mail: dermhjanaver.com

(c) Korean Society for Laser Medicine and Surgery

(@) This is an open access article distributed under the terms of the Creative Commons Attribution NonCommercial License (http://creativecommons.org/ licenses/by-nc/4.0) which permits unrestricted noncommercial use, distribution, and reproduction in any medium, provided the original work is properly cited.
}

\begin{abstract}
Partial unilateral lentiginosis (PUL) is a pigmentation disorder characterized by multiple lentigines covering the normal skin with a unilateral, segmented pattern that stops at the midline. There has only been a few successful PUL treatments reported in the literature, and an ideal therapeutic method has not yet been identified. We report a case of facial PUL that was successfully treated with a 532-nm Q-switched neodymium-ser with subsequent low-fluence 1,064-nm QS Nd:YAG laser treatment. The patient did not experience recurrence until 3 years after the treatment. This appears to be a promising therapeutic approach for PUL. Additional research should be conducted for further assessment of this method.
\end{abstract}

\section{Key words}

Partial unilateral lentiginosis; Lasers and light sources; Refractory 


\section{INTRODUCTION}

Partial unilateral lentiginosis (PUL) is a rare pigmentation disorder characterized by multiple lentigines that are grouped within an area of normal skin, often in a segmented pattern, that first appear at birth or in childhood.' As a consequence of its early appearance, PUL often compromises one or more dermatomes, and, with time, the lesions usually become darker and more extensive. ${ }^{2}$ Histologically, PUL exhibits lentiginous epidermal mela- nocyte proliferation and rete ridge elongation, similar to the simple lentigines. ${ }^{1}$ Although there is usually no concomitant abnormality, the disorder can occasionally be associated with abnormalities such as neurofibromatosis, café-au-lait spots, or ocular nevus. ${ }^{3}$

Only a few reports have studied laser treatment of PUL ${ }^{4,5}$ and optimal treatment guidelines have not yet been fully established. Q-switched (QS) neodymium-doped yttrium aluminum garnet ( $\mathrm{Nd}: Y A G$ ) has become a standard laser treatment for various pigmentation lesions because
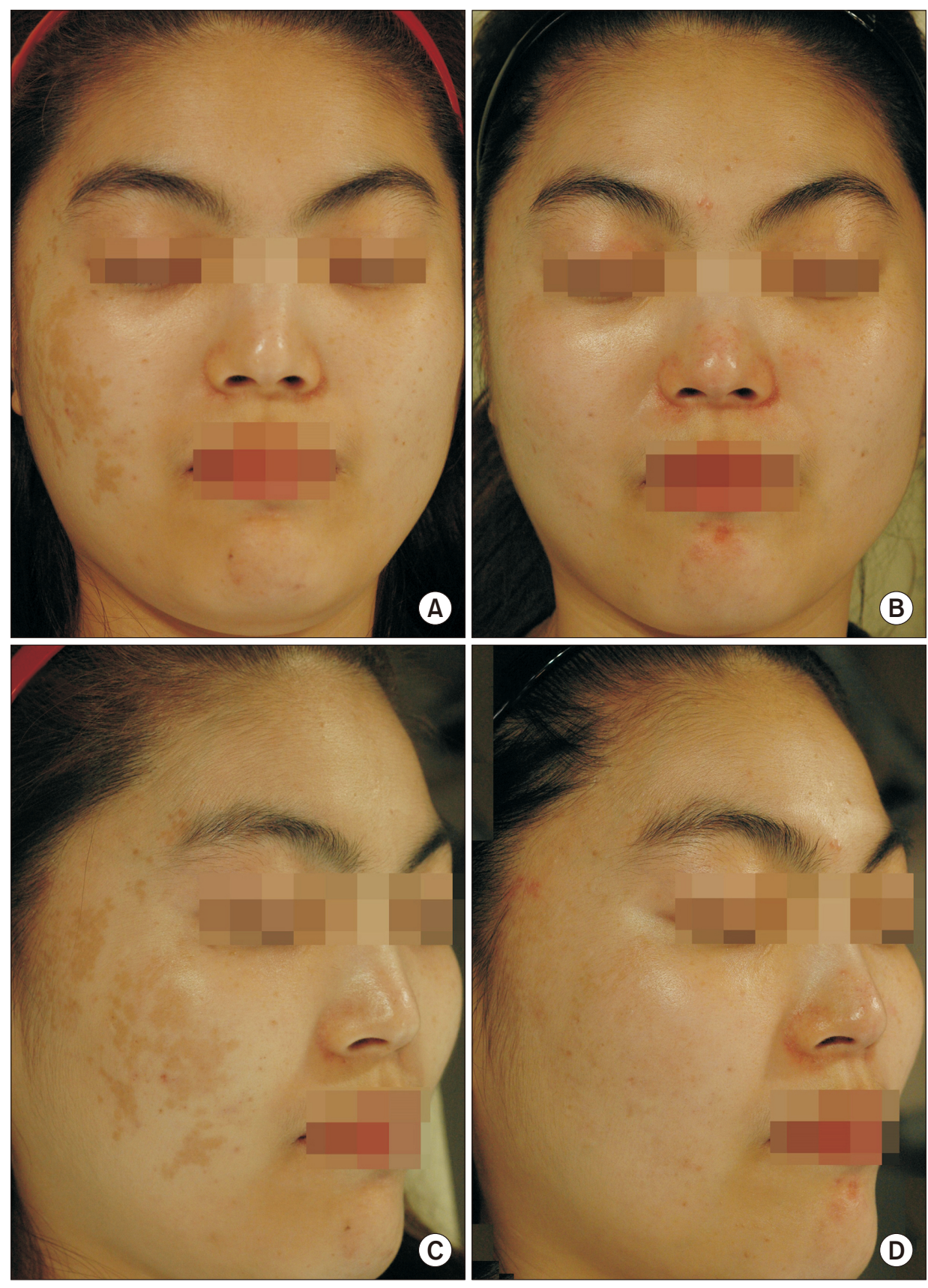

Fig. 1. A 22-year-old female patient with partial unilateral lentiginosis on the right cheek. Before the laser treatment ( $\mathrm{A}$ and $\mathrm{C}$ ) and 6 months after the last serial treatment (B and D) using 532-nm and subsequent 1,064-nm Q-switched neodymiumdoped aluminum garnet lasers. 
it causes highly selective melanin destruction. In this study, we present a case of facial PUL that was treated with 532-nm and subsequent low-fluenced 1,064-nm QS $\mathrm{Nd}$ :YAG lasers and had an excellent treatment response.

\section{CASE REPORT}

A 22-year-old Korean woman with skin phototype IV was transferred to our facility to manage a refractory facial pigment lesion that had persisted for approximately 10 years. She had previously received three QS alexandrite laser sessions at another hospital and the lesion had exhibited no response. Physical examination revealed numerous well-demarcated light brown macules and patches on the right side of the face in V2 and V3 terri- tories (Fig. 1A and C). There were no other concomitant mucocutaneous abnormalities, café au lait spots, axillary freckling, or neurofibromas. Neurological and ophthalmological examinations were all within normal range. She had no specific family medical history or underlying disease. Histopathologic examination showed hyperpigmentation of the lower epidermis, lentiginous elongation of rete ridges and an increased number of basal layer melanocytes. Immunohistochemical staining indicated an increased number of melanocytes (GP-100 stain) with increased basal pigmentation (Fontana-Masson stain) (Fig. 2). She was treated with a 532-nm QS Nd:YAG laser (SPECTRA $^{\circledR}$; LUTRONIC Corporation Ltd., Goyang, Korea), 4- to 5-mm spot size, using an energy fluence of $2.1-$ to $2.3-\mathrm{J} / \mathrm{cm}^{2}$ and a 5 - to $7-n$ pulse width at 3- to

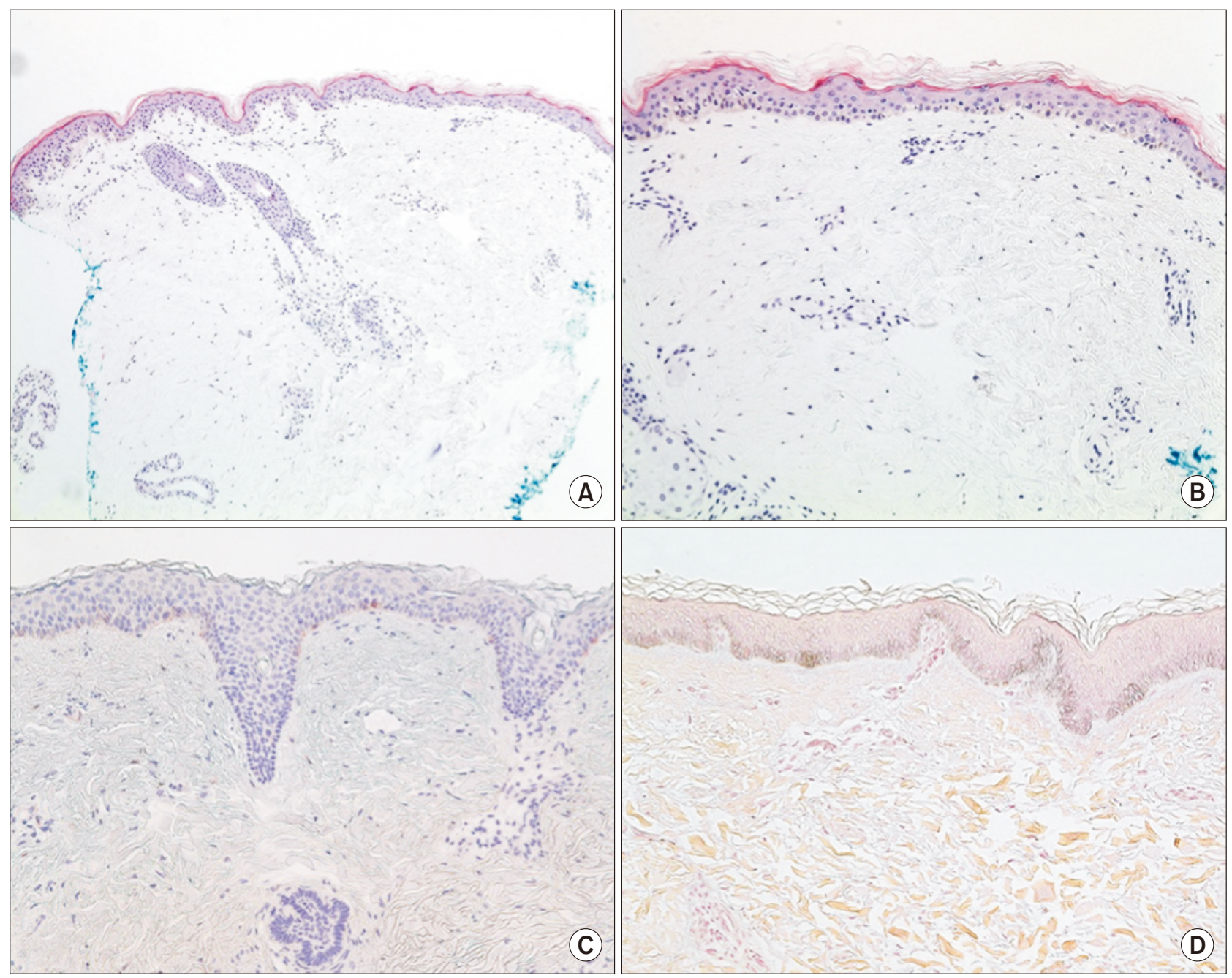

Fig. 2. Lentiginous elongation of rete ridges without the presence of dermal melanocytes (A) (hematoxylin and eosin, original magnification $\times 100)$. Hyperpigmentation of the basal layer and a slight increase in the number of melanocytes without nesting (B) (hematoxylin and eosin, original magnification $\times 200$ ). An increased number of melanocytes were identified on GP-100 staining $(C)$ and increased basal pigmentation was shown on Fontana-Masson staining (D) (original magnification $\times 200$ ). 
6-month intervals. This unusually long interval was due to the patient's personal preference, and five consecutive treatments were applied to the facial lesion. The facial PUL had an ongoing poor response (0-25\%) with fluence lower than $2.3 \mathrm{~J} / \mathrm{cm}^{2}$; however, the PUL had an excellent response (76\%-100\%) with a fluence of $2.3 \mathrm{~J} / \mathrm{cm}^{2}$ at the 5th treatment. Postinflammatory hyperpigmentation did not occur after 532-nm QS Nd:YAG laser treatment. The subtle remnant lesion was managed with five treatments with the 1064-nm QS Nd:YAG laser (SPECTRA ${ }^{\circledR}$ ), 8-mm spot size, using an energy fluence of $2.2 \mathrm{~J} / \mathrm{cm}^{2}$ at 2-week intervals. The lesion was almost completely resolved without any significant complications (Fig. 1B and D). No recurrence was reported until 3 years after the last treatment.

\section{DISCUSSION}

PUL is characterized by clustered unilateral lentigines on otherwise normal skin that histologically display features that are typical of lentigo. ${ }^{6}$ However, when 532-nm QS Nd:YAG was applied to PUL, similar to the treatment of lentigo, the rate of recurrence seemed very high according to the author's experience. PUL is benign and is not caused by other genetic disorders, such as PeutzJeghers syndrome or Leopard syndrome. PUL differs from these conditions in that it has a typically unilateral and segmentary distribution, and lacks all the clinical signs and symptoms found in these other syndromes. ${ }^{2,4}$ PUL has been associated with several cutaneous and systemic disorders including café-au-lait macules, nevus depigmentosus, segmental neurofibromatosis, mental retardation, seizures, sickle cell anemia, and celiac disease. Although the coexistence of different cutaneous and systemic disorders has occasionally been reported, the existence of skin lesions in patients with PUL is not an indication of the presence of other associated anomalies. ${ }^{7}$ Further evaluation is recommended to determine the possibility of an association with systemic involvement. ${ }^{4,5}$

PUL is characterized by the presence of pigmented epidermal lesions and therefore any treatment that damages the epidermis can improve epidermal pigment. ${ }^{5}$ However, non-invasive agents such as hydroquinone, tretinoin and glycolic acid showed inconsistent effects, and cryotherapy, which has been proven to be an effective treatment, can cause scarring and hypopigmentation. ${ }^{3}$ Even various laser treatments, based on the theory of selective photothermolysis, have shown inconsistent results and high recurrence rates. ${ }^{4,5}$ Theoretically, many laser modalities such as the 755-nm QS alexandrite laser and 532-nm and 1,064-nm QS Nd:YAG lasers could be used to treat PUL based on histologic findings. In fact, Pretel et al. reported positive outcomes using a Q-switched alexandrite laser for PUL treatment. ${ }^{5}$ However, the patient in our case was not responsive to the QS alexandrite laser even after three treatment sessions.

Epidermal pigmented lesions, such as freckles and solar lentigines, respond best to lasers with shorter wavelengths, such as 532-nm. ${ }^{8}$ Therefore, the 532-nm QS Nd:YAG laser was first applied to the PUL lesion. In comparison, dermal pigmented lesions, such as the nevus of Ota or acquired dermal melanosis, have conventionally been treated with longer wavelength lasers. The 1,064-nm QS Nd:YAG laser is often selected because of its longer wavelength, which provides relatively deep penetration into the skin, bypassing the epidermis to target aberrant dermal melanocytes with minimal injury to the surrounding normal skin. ${ }^{8}$ We designed a combined treatment, composed of 532-nm and subsequent 1,064-nm QS $\mathrm{Nd}$ :YAG laser therapy, to elicit a more complete PUL response, which was thought to be hindered by remnant pigment clusters in the deeper portions of the epidermis or pigment incontinence in the upper dermis. The PUL in our study was not responsive when the 2.1 - and $2.3-\mathrm{J} / \mathrm{cm}^{2}$ fluence of the 532-nm QS Nd:YAG laser was used, but we achieved a favorable result with the $2.3-\mathrm{J} / \mathrm{cm}^{2}$ fluence. Therefore, this could be used as a reference for both safety and efficacy to treat refractory epidermal pigmentation lesions such as PUL. The remaining PUL lesions were treated with subsequent low fluence 1,064-nm QS $\mathrm{Nd}$ :YAG lasers. This combination treatment is a promising therapeutic approach for PUL. Usually, PUL is responsive to 532-nm QS Nd:YAG laser treatment, exhibiting crust formation. However, high recurrence is a problem after the crust resolves. Although Lee et al. reported good response of PUL to low-fluence QS 1,064-nm Nd:YAG laser treatment, ${ }^{4}$ responses were gradual and multiple sessions (mean 6.6) were required. By combining 532-nm QS $\mathrm{Nd}$ :YAG laser treatment with low-fluence QS 1,064-nm $\mathrm{Nd}$ :YAG laser, prompt initial response and suppression of recurrence can be achieved, leading to fewer treatment sessions. We propose treating PUL with the 532-nm QS $\mathrm{Nd}$ :YAG laser followed by the 1,064-nm laser for more efficacious and complete results. Additional larger-scale and randomized controlled studies are necessary to verify and further investigate this method of treatment.

\section{REFERENCES}

1. Thompson GW, Diehl AK. Partial unilateral lentiginosis. Arch 
Dermatol 1980;116:356.

2. Schaffer JV, Lazova R, Bolognia JL. Partial unilateral lentiginosis with ocular involvement. J Am Acad Dermatol 2001;44(2 Suppl):387-90.

3. Serarslan G. Partial unilateral lentiginosis with ipsilateral ocular nevus. J Eur Acad Dermatol Venereol 2007;21:281-3.

4. Lee Y, Choi EH, Lee SW. Low-fluence Q-switched 1,064-nm neodymium-doped yttrium aluminum garnet laser for the treatment of facial partial unilateral lentiginosis in Koreans. Dermatol Surg 2012;38:31-7.

5. Pretel M, Irarrazaval I, Aguado L, Lera JM, Navedo M, Giménez de Azcárate A. Partial unilateral lentiginosis treated with alexandrite Q-switched laser: case report and review of the literature. J Cosmet Laser Ther 2013;15:207-9.

6. Trattner A, Metzker A. Partial unilateral lentiginosis. J Am Acad Dermatol 1993;29:693-5.

7. Romiti R, Harnache JDI, Neto CF, Kreuter A, Altmeyer P, Jansen $T$. Extensive partial unilateral lentiginosis. J Dermatol 2001;28:490-2.

8. Ee HL, Goh CL, Khoo LS, Chan ES, Ang P. Treatment of acquired bilateral nevus of ota-like macules (Hori's nevus) with a combination of the $532 \mathrm{~nm}$ Q-Switched Nd:YAG laser followed by the 1,064 nm Q-switched Nd:YAG is more effective: prospective study. Dermatol Surg 2006;32:34-40. 